\title{
The Impact of Exacerbation History on the Safety and Efficacy of Aclidinium in Patients with Chronic Obstructive Pulmonary Disease and Increased Cardiovascular Risk: ASCENT-COPD Trial
}

This article was published in the following Dove Press journal:

International Journal of Chronic Obstructive Pulmonary Disease

\author{
Robert A Wise $\mathbb{I D}^{\prime}$ \\ Kenneth R Chapman $\mathbb{D}^{2}$ \\ Benjamin M Scirica ${ }^{3,4}$ \\ Sami Z Daoud ${ }^{5}$ \\ Dan Lythgoe $\mathbb{D}^{6}$ \\ Esther Garcia-Gil ${ }^{7}$ \\ 'Medicine, Pulmonary and Critical Care, \\ Johns Hopkins University School of \\ Medicine, Baltimore, MD, USA; \\ ${ }^{2}$ Department of Medicine, University of \\ Toronto, Toronto, ON, Canada; \\ ${ }^{3}$ Cardiovascular Division, Brigham and \\ Women's Hospital, Boston, MA, USA; \\ ${ }^{4}$ Department of Medicine, Harvard \\ Medical School, Boston, MA, USA; ${ }^{5}$ Late- \\ Stage Respiratory and Immunology, \\ BioPharmaceuticals R\&D, AstraZeneca, \\ Gaithersburg, MD, USA; ${ }^{6}$ Statistics, \\ Phastar, Chiswick, London, UK; \\ ${ }^{7}$ Respiratory \& Immunology, \\ BioPharmaceuticals Medical, \\ AstraZeneca, Barcelona, Spain
}

Correspondence: Robert A Wise Medicine, Pulmonary and Critical Care, Johns Hopkins University School of Medicine, 550I Hopkins Bayview Circle, Baltimore, MD, 21224, USA

Email rwise@jhmi.edu
Purpose: Chronic obstructive pulmonary disease (COPD) exacerbations are associated with increased risk of major adverse cardiovascular events (MACE) and mortality. Here, we investigate whether the safety and efficacy of aclidinium bromide differ due to exacerbation history in patients with COPD and increased cardiovascular risk.

Patients and Methods: ASCENT-COPD was a Phase 4, multicenter, double-blind, randomized, placebo-controlled, parallel-group study of patients with moderate-to-very severe COPD and increased cardiovascular risk. Patients were randomized 1:1 to receive aclidinium or placebo twice daily for up to 3 years. Outcomes included time to first MACE and all-cause mortality over 3 years, exacerbation rate during the first year on-treatment, and change in baseline pre-dose forced expiratory volume in 1 second $\left(\mathrm{FEV}_{1}\right)$ over 3 years. This prespecified subgroup analysis compared outcomes in patients receiving aclidinium vs placebo. The comparison of patients with vs without an exacerbation history was added following a protocol amendment to increase enrollment in the primary study.

Results: Of 3589 patients, $2156(60.1 \%)$ had $\geq 1$ moderate or severe exacerbations in the prior year, compared with 1433 (39.9\%) without prior exacerbations. Although patients with an exacerbation history had numerically higher rates of MACE and mortality regardless of treatment, aclidinium did not increase risk of MACE $(\geq 1$ : hazard ratio [HR] $0.79,95 \%$ confidence interval [CI]: 0.54-1.16; none: HR 1.27, 95\% CI: $0.65-2.47$; interaction $P=0.233$ ) or all-cause mortality ( $\geq 1$ : HR 1.08, 95\% CI: $0.81-1.43$; none: HR $0.66,95 \%$ CI: $0.36-1.22$; interaction $P=0.154$ ), regardless of exacerbation history. Aclidinium reduced the exacerbation rate vs placebo irrespective of exacerbation history ( $\geq 1$ : rate ratio [RR] $0.80,95 \% \mathrm{CI}$ : 0.68-0.94; none: RR 0.69, 95\% CI: 0.54-0.89; interaction $P=0.340$ ) and improved $\mathrm{FEV}_{1}$ (interaction $P=0.633$ ).

Conclusion: In patients with moderate-to-very severe COPD and increased cardiovascular risk, aclidinium did not increase risk of MACE or mortality and reduced exacerbation rate vs placebo, regardless of exacerbation history.

Clinical Trial Registration: ClinicalTrials.gov Identifier: NCT01966107.

Keywords: COPD, COPD exacerbation, aclidinium, MACE, mortality

\section{Introduction}

Chronic obstructive pulmonary disease (COPD) is a heterogeneous disease characterized by persistent respiratory symptoms and airflow obstruction and is a leading cause 
of morbidity and mortality worldwide. ${ }^{1}$ In a review of five studies, over $70 \%$ of patients experienced $\geq 1$ exacerbations within three years of study initiation, ${ }^{2}$ and these events play a substantial role in the disease burden for both patients and healthcare systems. Exacerbations can be characterized as mild (requiring an increase in dose of regular medication), moderate (requiring additional medication from a physician, such as corticosteroids and/or antibiotics), or severe (resulting in hospitalization and/or mortality). ${ }^{3}$ Exacerbations are associated with an accelerated decline in lung function ${ }^{4,5}$ and an increased risk of major adverse cardiovascular events (MACE; defined as cardiovascular mortality, non-fatal myocardial infarction, or non-fatal stroke $)^{6-9}$ and mortality. ${ }^{10-12}$

Aclidinium bromide $400 \mu \mathrm{g}$ twice daily is a long-acting muscarinic antagonist approved for use as maintenance treatment for patients with COPD, administered using a breath-actuated dry-powder inhaler (Genuair/Pressair; AstraZeneca). ${ }^{13,14}$ ASCENT-COPD was a Phase 4, multicenter, double-blind, randomized, placebo-controlled, parallel-group study that evaluated cardiovascular $(\mathrm{CV})$ safety and COPD exacerbation rate in patients with moderate-to-very severe COPD and increased CV risk. ${ }^{15}$ Treatment with aclidinium did not increase risk of MACE vs placebo over 3 years and reduced the rate of COPD exacerbations vs placebo over one year. ${ }^{15}$

Most studies that aim to reduce COPD exacerbation risk enroll patients with a history of exacerbations and exclude patients with CV risk factors. ${ }^{3,16,17}$ ASCENT-COPD provided a unique population of patients with or without an exacerbation history and an increased CV risk. This pre-specified subgroup analysis aimed to compare the effect of aclidinium treatment on MACE, exacerbations, and lung function in ASCENT-COPD patients. The comparison of patients with vs without an exacerbation history was added following a protocol amendment to increase enrollment in the primary study. The hypothesis of this subgroup analysis was that a benefit in exacerbation prevention with aclidinium vs placebo would not be found in patients without an exacerbation history, but aclidinium would demonstrate improved lung function vs placebo. Moreover, it was hypothesized that patients with an exacerbation history would have more MACE and COPD exacerbations compared with patients without an exacerbation history.

\section{Methods}

\section{Study Design}

The study design for the ASCENT-COPD study has been reported in detail previously. ${ }^{18}$ In brief, ASCENT-COPD (NCT01966107) was a Phase 4, multicenter, double-blind, randomized, placebo-controlled, parallel-group study conducted at 522 sites in the USA and Canada. ${ }^{15}$ The study comprised a 2 -week washout period, followed by a doubleblind treatment phase, during which patients were randomized 1:1 to receive aclidinium $400 \mu \mathrm{g}$ or matching placebo twice daily for up to 3 years, until $\geq 122$ MACE events occurred. In this subgroup analysis, patients were categorized according to whether or not they had experienced $\geq 1$ moderate or severe COPD exacerbations (requiring additional medication from a physician, such as corticosteroids and/or antibiotics, or resulting in hospitalization and/or mortality) in the year prior to the study.

\section{Study Population}

The study population for the ASCENT-COPD study has been previously reported. ${ }^{15}$ In brief, eligible patients were males or females aged $\geq 40$ years with moderate-to-very severe COPD (forced expiratory volume in 1 second $\left[\mathrm{FEV}_{1}\right] /$ forced vital capacity $<70 \%$, and $\mathrm{FEV}_{1}<80 \%$ predicted) and a smoking history of $\geq 10$ pack-years. Details of the $\mathrm{CV}$ risk factors can be found in the supplementary materials. When the ASCENT-COPD study was started, patients were required to have had $\geq 1$ treated $C O P D$ exacerbations in the year prior to screening; however, this requirement was subsequently removed after approximately half of the patients were enrolled to increase accrual and allow for a broader patient population. At that time, the upper limit of $\mathrm{FEV}_{1}$ was also increased from $70 \%$ to $80 \%$ predicted.

\section{Outcome Measures}

Safety outcomes included time to first MACE over 3 years, as adjudicated by a clinical endpoint adjudication committee, ${ }^{15}$ and all-cause mortality. Efficacy outcomes were annual rate of moderate or severe COPD exacerbations during the first year on-treatment and change in baseline pre-dose (trough) $\mathrm{FEV}_{1}$ over 3 years.

\section{Statistical Considerations}

All statistical analyses were based on the full analysis set (all patients who took $\geq 1$ dose of treatment), and patients were analyzed according to their randomized treatment. Safety analyses included "on-study" data, ie, all data collected during the follow-up period, irrespective of treatment discontinuation. Efficacy analyses included "on-treatment" data, ie, data up to discontinuation of randomized treatment, where applicable; MACE and moderate or severe COPD exacerbation rate analyses were pre-specified. 
Time to first MACE was analyzed using subgroupspecific (ie, no or $\geq 1$ exacerbations) Cox proportional hazards models with randomized treatment group (aclidinium/placebo), history of $\geq 1$ exacerbation in the previous year (yes/no), baseline CV risk group (prior events and risk factors), and smoking status (current smoker, ex-smoker) as factors. To assess evidence of a differential treatment effect, an additional analysis was undertaken, including both subgroups and a treatment-byexacerbation history interaction term. This approach was also undertaken for all-cause mortality. Moderate or severe COPD exacerbation rates were analyzed using negative binomial regression models with treatment group and exacerbation history, and their two-way interaction. Spirometry outcomes were analyzed on-treatment during the first year using mixed models for repeated measures adjusted for pre- and post-bronchodilator $\mathrm{FEV}_{1}$ at screening, baseline $\mathrm{FEV}_{1}$, smoking status, baseline inhaled corticosteroid use, exacerbation history, and visits. In addition, this included a three-way interaction between treatment, baseline exacerbation history, and study visit.

To compare the incidence of MACE and all-cause mortality between those with and without exacerbation history it was necessary to account for differential followup times between these subgroups. Cox regression models including randomized treatment group, history of $\geq 1$ exacerbation in the previous year, baseline $\mathrm{CV}$ risk group, smoking status and the interaction between exacerbation history and treatment group were used. The overall effect of exacerbation history was obtained by averaging the treatment-specific effect estimates.

All reported outputs were produced using SAS version 9.3 (SAS Institute Inc). $P$ values for treatment-byexacerbation history interaction effects were considered statistically significant if $P<0.10$; otherwise $P$ values less than 0.05 were considered statistically significant.

\section{Results}

\section{Baseline Demographics and Characteristics}

Of the 3589 patients included in this analysis, 2156 (60.1\%) patients had a history of $\geq 1$ moderate or severe COPD exacerbations in the year prior to randomization compared with 1433 (39.9\%) patients without prior COPD exacerbations (Figure 1). Baseline demographics were generally comparable between patients with and without an exacerbation history; however, baseline COPD characteristics and CV risk differed (Table 1). Patients with an exacerbation history had worse airflow obstruction (14.9\% very severe, $44.1 \%$ severe, $39.1 \%$ moderate, and $0.5 \%$ mild) vs patients without an exacerbation history $(10.0 \%$ very severe, $35.2 \%$ severe, $53.1 \%$ moderate, and $0.4 \%$ mild). A higher percentage of patients with vs without an exacerbation history had one or more CV events, plus two or more atherothrombotic risk factors $(47.3 \%$ vs $38.9 \%$, respectively). Prior inhaled corticosteroid use was greater in patients with vs without an exacerbation history, both alone and in combination with a long-acting $\beta_{2}$-agonists and/or long-acting muscarinic antagonist (60.0\% vs $51.9 \%$, respectively). COPD assessment test total and individual scores were similar between patients with and without an exacerbation history (total score, mean [standard deviation, SD]: 21.0 [7.3] vs 20.2 [7.1], respectively). Patients with an exacerbation history had lower lung function vs patients without an exacerbation history (post-bronchodilator $\mathrm{FEV}_{1} \%$ predicted, mean [SD] 45.7 [14.6] vs 50.7 [15.1], respectively).

\section{Safety}

Although patients with an exacerbation history experienced numerically more MACE than patients without an exacerbation history ( $\geq 1$ exacerbation: $5.1 \%$; no exacerbations: $2.4 \%$; hazard ratio [HR] 1.34, 95\% confidence intervals [CI] $0.90-2.00 ; P=0.156$ ), aclidinium did not increase risk of MACE, regardless of exacerbation history $(\geq 1$ exacerbation: HR $0.79,95 \%$ CI: $0.54-1.16$; no exacerbations: HR 1.27, 95\% CI: $0.65-2.47$; interaction $P=0.233$; Figure 2).

The rate of all-cause mortality was numerically higher in patients with an exacerbation history $(\geq 1$ exacerbation: $8.9 \%$; no exacerbations: $2.9 \%$; HR $1.11,95 \%$ CI $0.76-$ $1.62 ; P=0.580)$. However, aclidinium did not increase allcause mortality risk, irrespective of COPD exacerbation ( $\geq 1$ exacerbation: HR 1.08, 95\% CI: 0.81-1.43; no exacerbations: HR $0.66,95 \%$ CI: $0.36-1.22$; interaction $P=0.154$; Figure 3).

\section{Efficacy}

The hazard ratios for time to first moderate or severe COPD exacerbations (Figure 4A) were similar for patients with or without an exacerbation history (interaction $P=0.279)$. The annual exacerbation rate was numerically higher in patients who had a history of COPD exacerbations ( $\geq 1$ exacerbation: aclidinium 0.65 vs placebo 0.82 , absolute rate reduction: 0.17 ; no exacerbation: aclidinium 


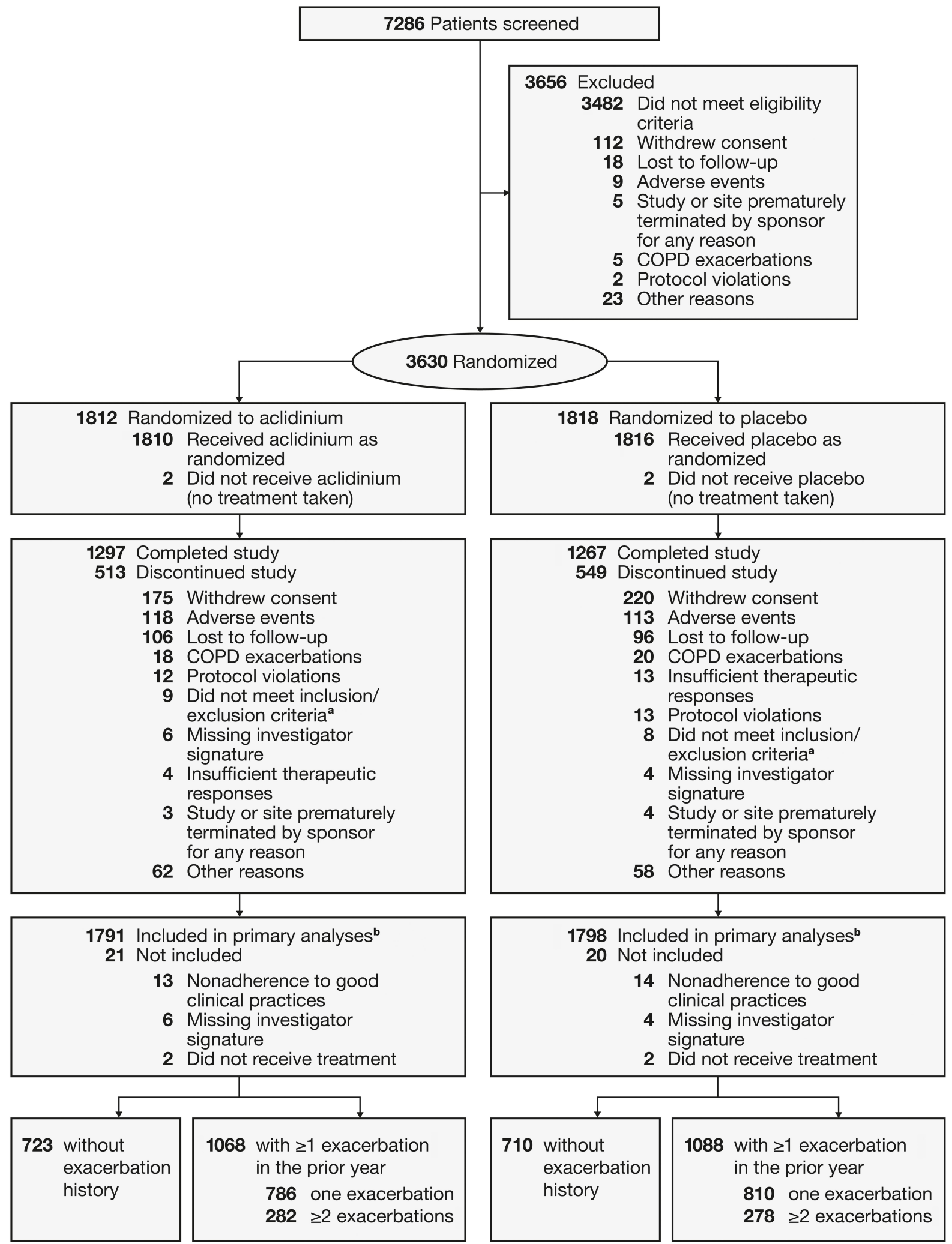

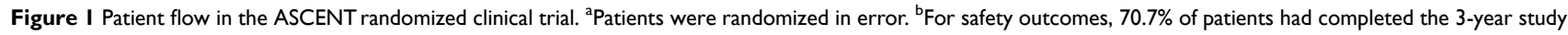
or were currently enrolled in the study when it was stopped; for efficacy outcomes, $67.3 \%$ of patients had completed 12 months of treatment or were in their first year of treatment when the study was stopped. The median exposure times for aclidinium vs placebo were: 770.0 and 736.5 days with a history of $\geq 1$ exacerbation and 410.0 and 386.0 days without exacerbation history, respectively.

Abbreviation: COPD, chronic obstructive pulmonary disease. 
Table I Baseline Demographics and Characteristics

\begin{tabular}{|c|c|c|}
\hline Patients & $\begin{array}{l}\geq \text { I Exacerbations in Previous } \\
\text { Year } \\
\text { Total }(N=2 \text { I 56) }\end{array}$ & $\begin{array}{l}\text { No Exacerbations in Previous } \\
\text { Year } \\
\text { Total }(\mathrm{N}=1433)\end{array}$ \\
\hline Mean age, years (SD) & $66.9(8.3)$ & $67.5(8.5)$ \\
\hline Male, \% & 57.6 & 60.3 \\
\hline White, \% & 90.9 & 90.2 \\
\hline Current smoker, \% & 42.8 & 44.7 \\
\hline CAT total score, mean (SD) & $21.0(7.3)$ & $20.2(7.1)$ \\
\hline Cough score, mean (SD) & $2.7(1.2)$ & $2.7(1.2)$ \\
\hline Phlegm score, mean (SD) & $2.6(1.3)$ & $2.4(1.3)$ \\
\hline Chest feeling tight, mean (SD) & $2.1(1.4)$ & $1.9(1.3)$ \\
\hline Breathless going up, mean (SD) & $3.8(1.2)$ & $3.7(1.2)$ \\
\hline Feeling limited, mean (SD) & $2.8(1.5)$ & $2.6(1.4)$ \\
\hline Confident leaving home, mean (SD) & $1.6(1.5)$ & $1.6(1.4)$ \\
\hline Sound sleep, mean (SD) & $2.4(1.5)$ & $2.3(1.5)$ \\
\hline Energy level, mean (SD) & $3.1(1.3)$ & $3.0(1.2)$ \\
\hline Post-bronchodilator $\mathrm{FEV}, \%$ predicted, mean (SD) & $45.7(14.6)$ & $50.7(15.1)$ \\
\hline \multicolumn{3}{|l|}{ COPD exacerbations in previous year, $\mathbf{n}(\%)$} \\
\hline $\mathbf{0}$ & 0 & $1433(100.0)$ \\
\hline $\mathbf{I}$ & $1596(74.0)$ & 0 \\
\hline$\geq 2$ & $560(26.0)$ & 0 \\
\hline COPD exacerbation rate in previous year, mean (SD) & I.4 (0.9) & 0.0 \\
\hline \multicolumn{3}{|l|}{ COPD severity based on airflow obstruction, $n$ (\%) } \\
\hline Mild & II $(0.5)$ & $6(0.4)$ \\
\hline Moderate & $843(39.1)$ & $761(53.1)$ \\
\hline Severe & $951(44.1)$ & $505(35.2)$ \\
\hline Very severe & $321(14.9)$ & $143(10.0)$ \\
\hline \multicolumn{3}{|l|}{ CV risk factors, \% } \\
\hline$\geq \mathbf{I}$ prior $\mathrm{CV}$ event $+\geq 2$ atherothrombotic risk factors & 47.3 & 38.9 \\
\hline$\geq \mathrm{I}$ prior CV event only & 3.5 & 4.3 \\
\hline$\geq 2$ atherothrombotic risk factors only & 48.9 & 56.6 \\
\hline
\end{tabular}

Notes: Full analysis set $(\mathrm{N}=3589)$; included all patients randomized to treatment who received $\geq \mathrm{I}$ dose of study drug. COPD severity was defined according to percent predicted FEV (GOLD I, mild, FEV I $\geq 80 \%$; GOLD 2, moderate, $50 \% \leq F E V_{1}<80 \%$; GOLD 3, severe, $30 \% \leq F E V_{1}<50 \%$; GOLD 4, very severe, FEV $<30 \%$ ).

Abbreviations: CAT, COPD assessment test; COPD, chronic obstructive pulmonary disease; $\mathrm{CV}$, cardiovascular; $\mathrm{FEV}_{\mathrm{l}}$, forced expiratory volume in I second; $\mathrm{n}$, number of patients; N, total number of patients; SD, standard deviation.

0.27 vs placebo 0.38 , absolute rate reduction: 0.11; Figure 4B). Aclidinium reduced the annual exacerbation rate vs placebo for patients with or without an exacerbation history with a similar relative benefit ( $\geq 1$ exacerbation: rate ratio [RR] 0.80, 95\% CI: 0.68-0.94; no exacerbations: RR 0.69 , 95\% CI $0.54-0.89$; interaction $P=0.340$ ). In addition, benefits of treatment with aclidinium in reducing exacerbation rate were irrespective of whether the exacerbation required systemic corticosteroid or antibiotic use (of note, patients could be counted in $>1$ category; Figure 4C); RRs were comparable between the two exacerbation treatment approaches (systemic corticosteroid: RR 0.81, 95\% CI: 0.70-0.95; antibiotics: RR 0.75, 95\% CI 0.65-0.87).
In terms of lung function, trough $\mathrm{FEV}_{1}$ was higher in patients with or without an exacerbation history treated with aclidinium vs placebo (Figure 5). Although treatment differences were numerically higher for patients with an exacerbation history vs those without (least square mean difference $93 \mathrm{~mL}$, 95\% CI: 66-120 vs $65 \mathrm{~mL}$, 95\% CI: 27-103), there was no evidence of a differential effect of treatment across subgroups (interaction $P=0.633$ ).

\section{Discussion}

In this subgroup analysis of the ASCENT-COPD study of patients with moderate-to-very severe COPD and $\mathrm{CV}$ risk factors, the risk of MACE, all-cause mortality, and rate of 

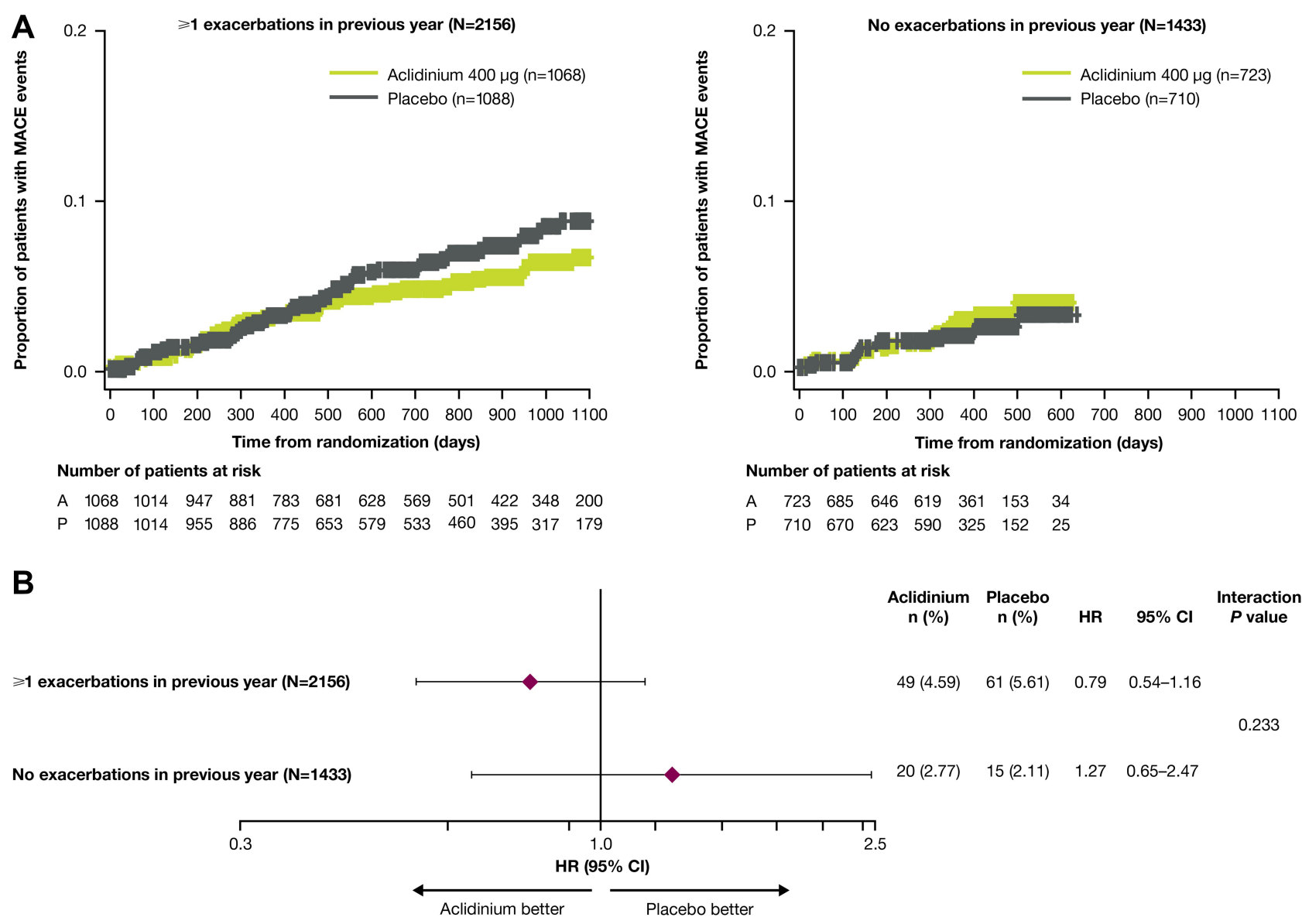

Figure 2 Time to first adjudicated MACE (A) and risk of MACE (B) up to 3 years in patients with and without an exacerbation history. Cox regression model with factors, including treatment, exacerbation history, and their interaction, as well as adjusting for other baseline factors (see Statistical Considerations). An HR $>I$ indicated higher risk of MACE with aclidinium and an $\mathrm{HR}<1$ indicated lower risk of MACE with aclidinium.

Abbreviations: $\mathrm{A}$, aclidinium; $\mathrm{Cl}$, confidence interval; $\mathrm{HR}$, hazard ratio; $\mathrm{MACE}$, major adverse cardiovascular event; $\mathrm{n}$, number of patients with $\mathrm{MACE}$; $\mathrm{N}$, total number of patients; P, placebo.

moderate-to-very severe COPD exacerbations were found to be numerically higher in patients with an exacerbation history vs those without an exacerbation history. Importantly, the safety of aclidinium with regards to MACE and all-cause mortality was similar to placebo, even in the higher risk population with a COPD exacerbation history. Although the relative reduction in rate of COPD exacerbations was similar in patients regardless of exacerbation history, the absolute reduction in exacerbation rate was greater in patients with an exacerbation history vs those without an exacerbation history. Improvements in pre-dose $\mathrm{FEV}_{1}$ were observed in all patients treated with aclidinium vs placebo, regardless of exacerbation history.

Overall and similar to previous observations, ${ }^{6-9,11,12}$ patients with a history of COPD exacerbation had a numerically increased risk of MACE and all-cause mortality vs those without an exacerbation history.
In a retrospective analysis of the UPLIFT trial, which evaluated symptoms, lung function, and exacerbation history, ${ }^{19}$ patients in GOLD Groups A and B, had exacerbations with tiotropium (RR 0.64 and 0.72 , respectively) that were similar to the non-exacerbation subgroup in this analysis (RR 0.69) in this study; however, in GOLD Groups $\mathrm{C}$ and $\mathrm{D}$, exacerbations with tiotropium (RR 0.91 and 0.89 , respectively) were numerically higher than the prior-exacerbation subgroup reported here (RR 0.80). Rates of MACE were similar between tiotropium and placebo for GOLD Groups $\mathrm{A}-\mathrm{C}$ in the UPLIFT trial; however, tiotropium significantly reduced the rate of MACE in GOLD Group D vs placebo. Because of the inclusion of patients with an increased CV risk in the ASCENT-COPD study, and subgroup definitions, it is not possible to say whether aclidinium is more effective than tiotropium in patients with more severe COPD (ie, patients in GOLD Groups 

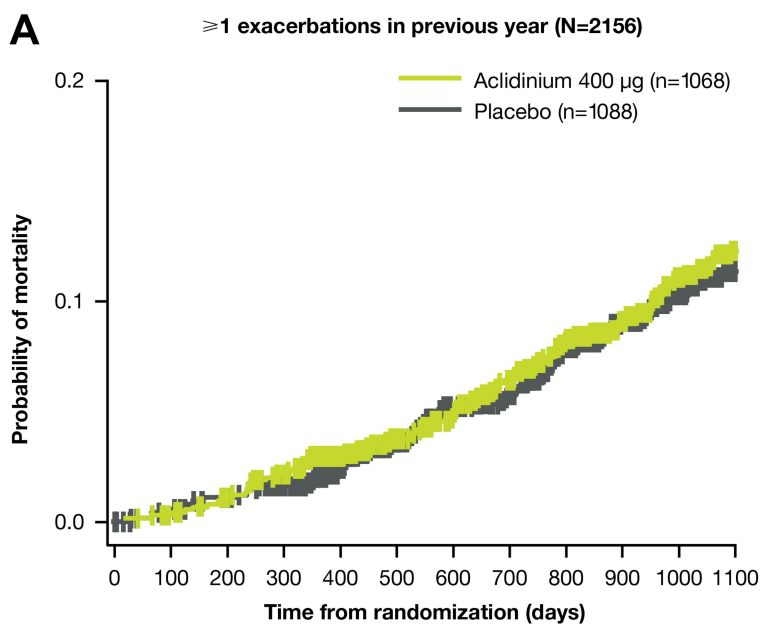

Number of patients at risk

$\begin{array}{lllllllllllll}\text { A } & 1068 & 1057 & 1037 & 1013 & 923 & 828 & 775 & 723 & 659 & 561 & 487 & 308\end{array}$

$\begin{array}{lllllllllllll}\text { P } & 1088 & 1072 & 1051 & 1031 & 935 & 823 & 759 & 723 & 652 & 570 & 475 & 288\end{array}$
No exacerbations in previous year $(\mathrm{N}=1433)$

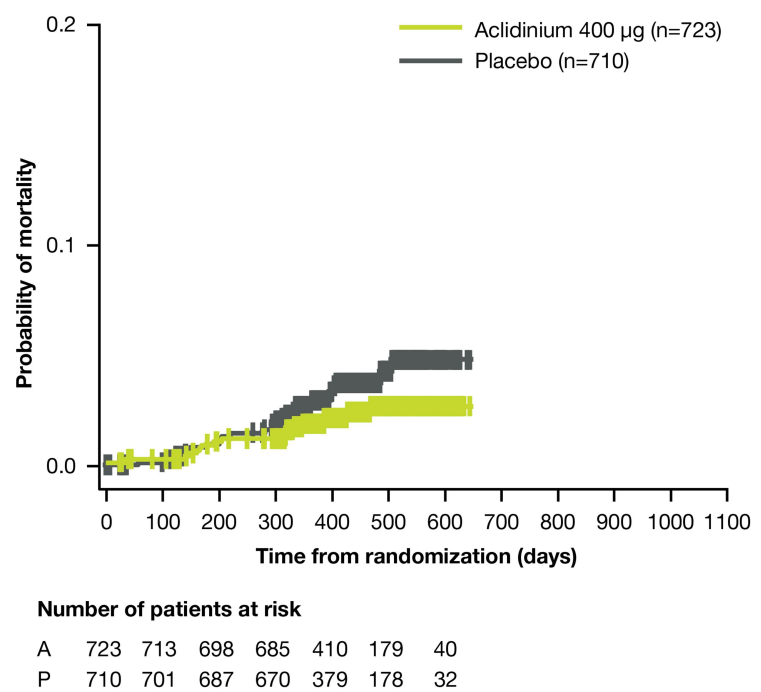

B

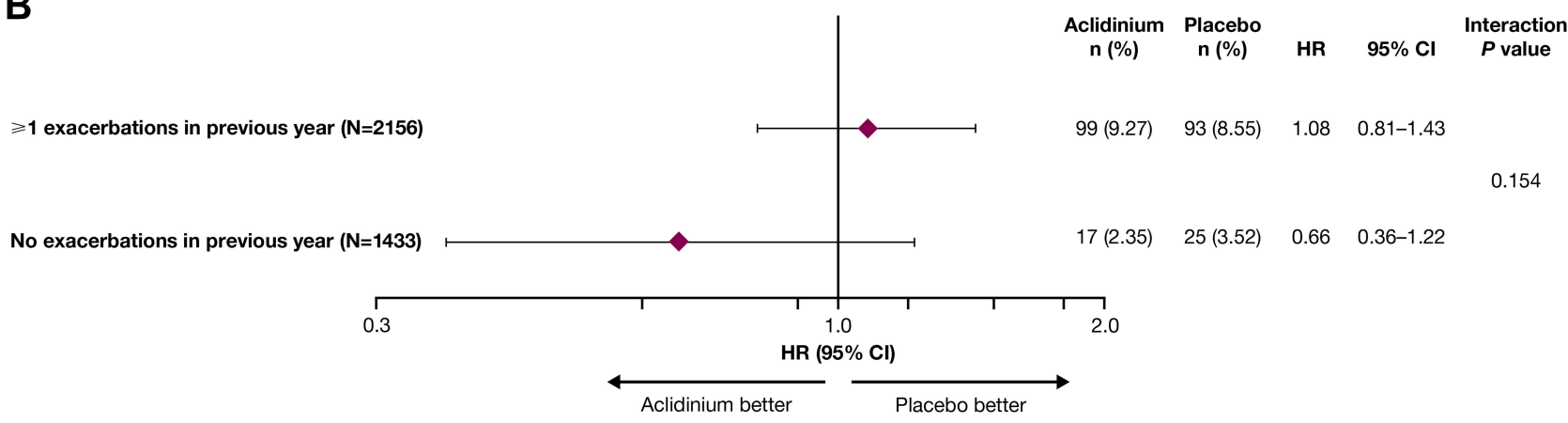

Figure 3 Time to all-cause mortality event (A) and risk of all-cause mortality (B) up to 3 years based on vital status in patients with and without an exacerbation history. Cox regression model with factors, including treatment, exacerbation history, and their interaction, as well as adjusting for other baseline factors (see Statistical Considerations). An HR $>I$ indicated higher risk of all-cause mortality with aclidinium and an $\mathrm{HR}<\mathrm{I}$ indicated lower risk of all-cause mortality with aclidinium.

Abbreviations: A, aclidinium; $\mathrm{Cl}$, confidence interval; HR, hazard ratio; $\mathrm{n}$, number of patients who died; $\mathrm{N}$, total number of patients; $\mathrm{P}$, placebo.

$\mathrm{C}$ and D, and those with an exacerbation history). However, coupled with results from the UPLIFT trial, these findings support and extend those of the overall ASCENT-COPD study ${ }^{15}$ and increase the confidence that long-acting muscarinic antagonists do not confer increased risk of $\mathrm{CV}$ disease in patients with COPD. ${ }^{19}$

Strengths of the study include the use of prospective and uniform data collection and verified definitions of both exacerbations and MACE. Furthermore, in contrast to observational studies, the ASCENT-COPD study was designed to demonstrate the effects of aclidinium, including the rate of exacerbations, in patients with COPD and increased $\mathrm{CV}$ risk, and as such the treatment assignment to aclidinium was random, regardless of COPD exacerbation history.

Limitations of the study include the change in patient enrollment after the study had begun; although an exacerbation history was an inclusion factor at the beginning of the study, the enrollment of patients without an exacerbation history was permitted from approximately half-way through the study, therefore the two subpopulations were not completely concurrent. Together with study completion at 122 MACE events, this led to a longer follow-up time for patients with a COPD exacerbation history with respect to MACE and mortality; however, comparison of treatment effects between subgroups remain valid. In the ASCENT-COPD study, aclidinium was shown to be non-inferior to placebo for MACE risk. Finally, exacerbation history in the year prior to recruitment was ascertained via taking patient history, and therefore was subject to recall bias and uncertainty; COPD exacerbations are frequently under-reported $^{20}$ and some overlap in exacerbation history between groups cannot be excluded. 

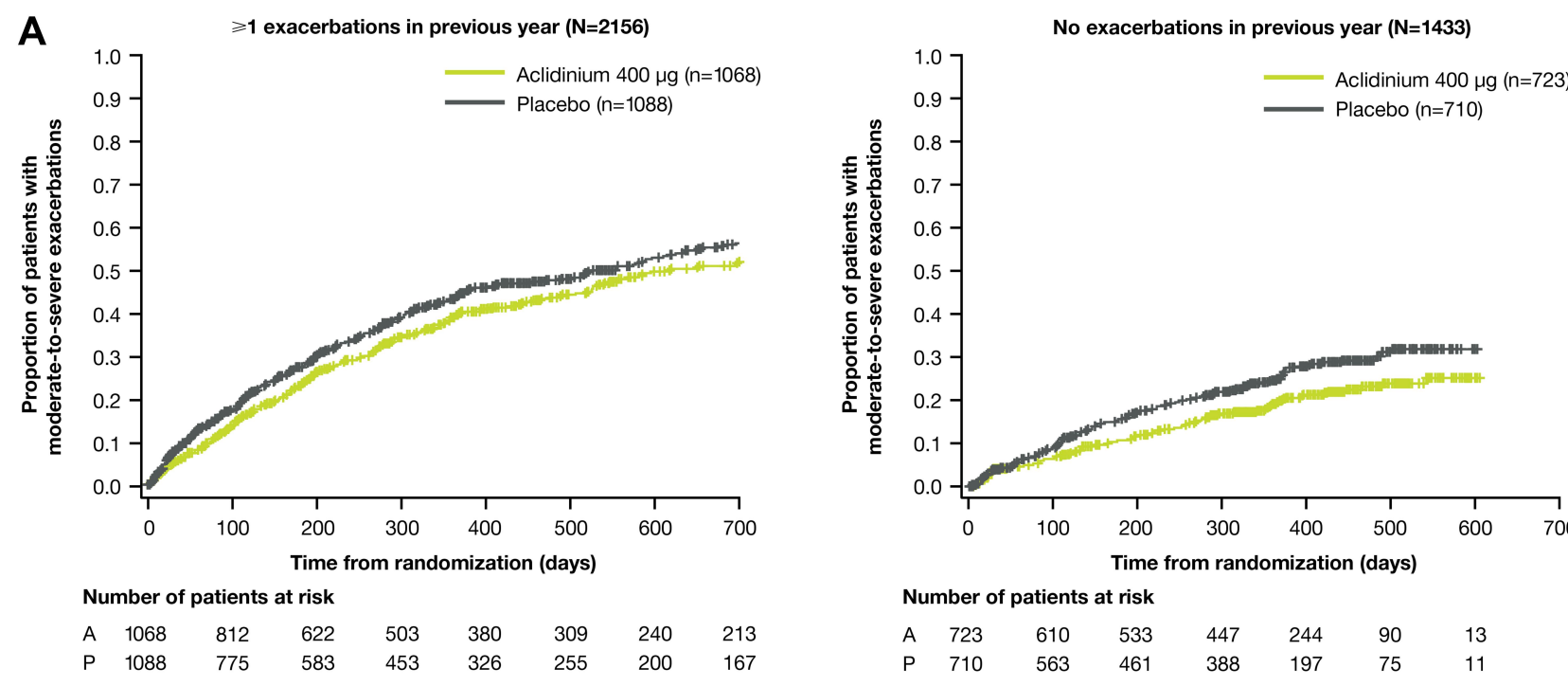

B

$\geqslant 1$ exacerbations in previous year $(\mathrm{N}=2156)$

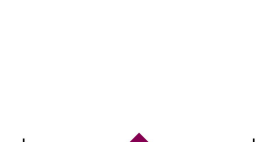

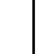

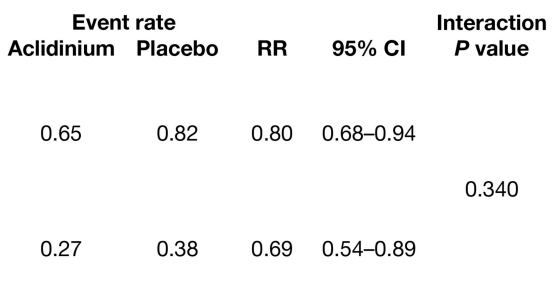

No exacerbations in previous year $(\mathrm{N}=1433)$
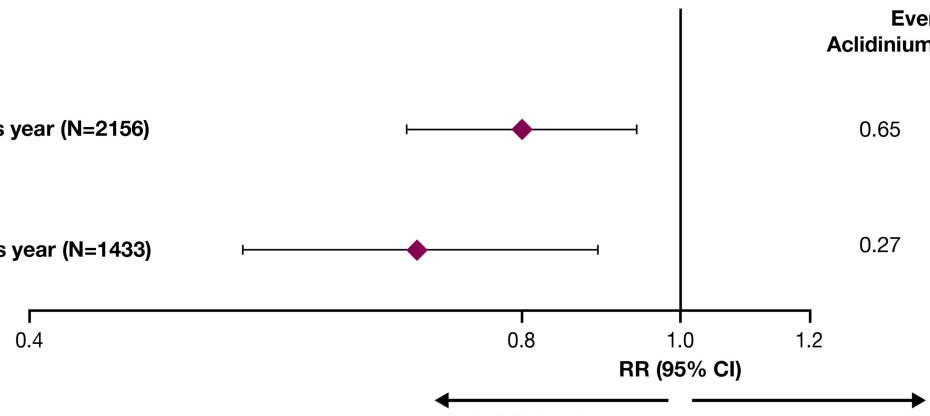

Aclidinium better Placebo better

C

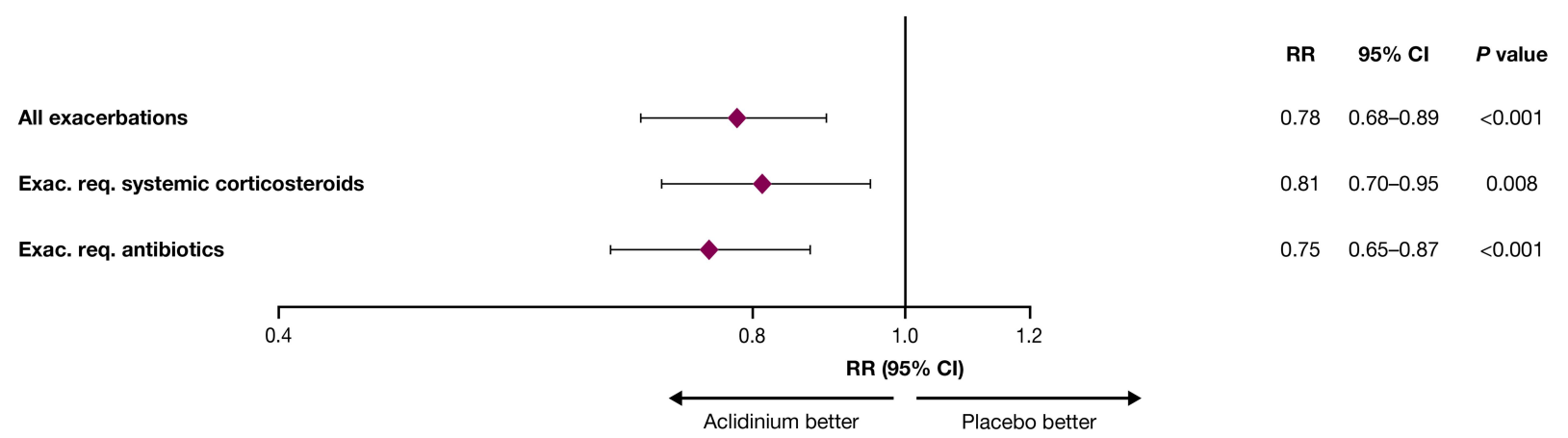

Figure 4 Time to first moderate or severe COPD exacerbation (A), moderate or severe COPD exacerbation rate during the first year (B), and COPD exacerbation treatments (C) (on-treatment analysis). Negative binomial model with factors, including treatment, exacerbation history, and their interaction. An RR $>$ I indicated higher risk of exacerbation with aclidinium and an $R R<1$ indicated lower risk of exacerbation with aclidinium.

Abbreviations: A, aclidinium; $\mathrm{Cl}$, confidence interval; COPD, chronic obstructive pulmonary disease; $\mathrm{N}$, total number of patients; $\mathrm{n}$, number of patients receiving specified treatment; P, placebo; RR, rate ratio.

\section{Conclusion}

In this pre-specified subgroup analysis of the ASCENT-

COPD study of patients with moderate-to-very severe COPD and increased CV risk, patients with a history of COPD exacerbation were found to have increased $\mathrm{CV}$ risk, compared with patients who had no prior exacerbations. However, there was no safety signal to suggest increased risk of MACE or mortality due to aclidinium vs placebo, irrespective of exacerbation history. In addition, treatment with aclidinium reduced the rate of COPD 

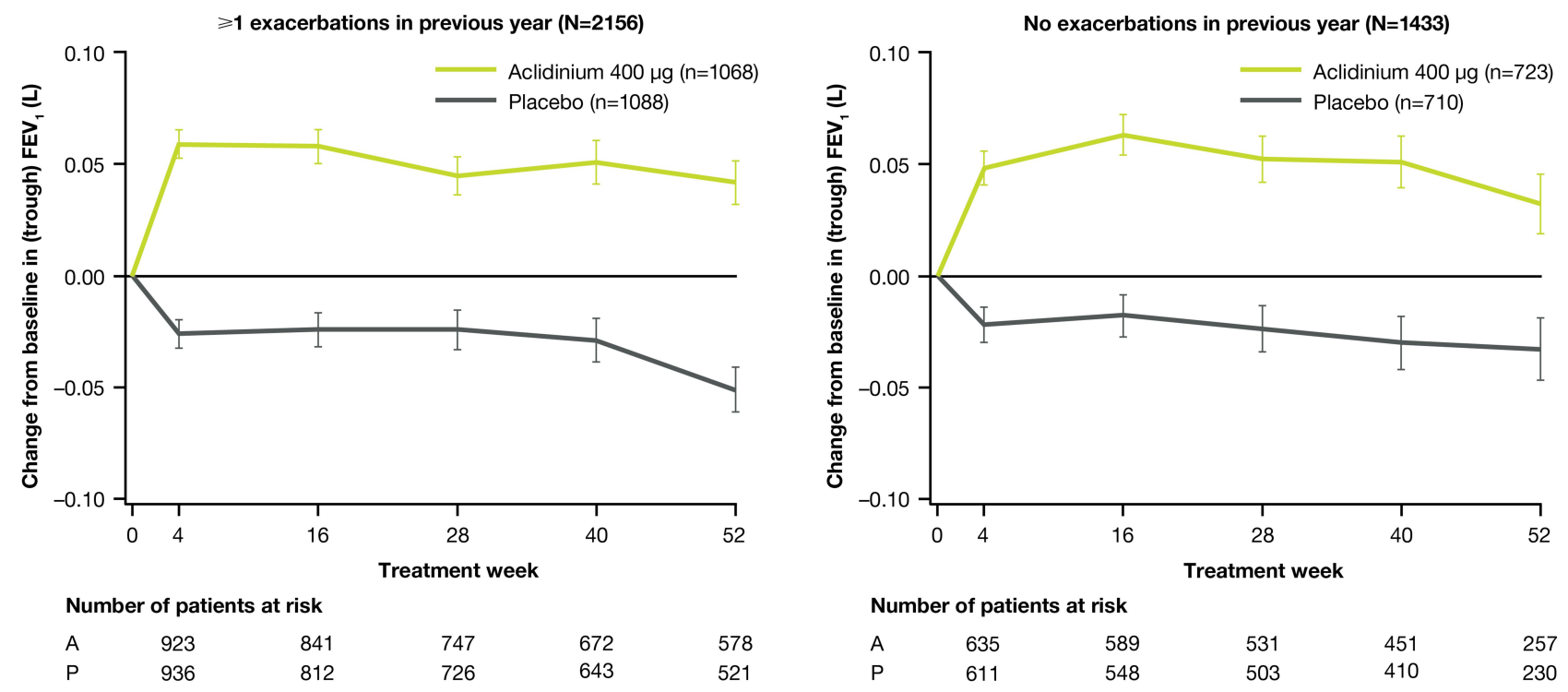

Figure 5 Change from baseline in morning (trough) FEV , during the first year (on-treatment analysis). Baseline = average of two pre-dose values prior to administration of first dose, or one value if only one is available, or pre-dose bronchodilator value at screening if both are missing. Change in baseline FEV (least square mean \pm standard error) analysis is based on a mixed model for repeated measures with pre- and post-bronchodilator FEV, values at screening, and baseline FEV , as factors. On-treatment analysis included all patients who completed I year or were on-treatment when the study was stopped.

Abbreviations: A, aclidinium; $\mathrm{FEV}_{1}$, forced expiratory volume in I second; $\mathrm{N}$, total number of patients; $\mathrm{n}$, number of patients receiving specified treatment; $\mathrm{P}$, placebo.

exacerbations in patients, regardless of exacerbation history.

Aclidinium may be used to prevent future COPD exacerbations in patients with moderate-to-very severe COPD and increased $\mathrm{CV}$ risk without increased risk of MACE or mortality, regardless of exacerbation history.

\section{Abbreviations}

A, aclidinium; CAT, COPD assessment test; CI, confidence interval; COPD, chronic obstructive pulmonary disease; $\mathrm{CV}$, cardiovascular; $\mathrm{FEV}_{1}$, forced expiratory volume in 1 second; HR, hazard ratio; MACE, major adverse cardiovascular event; $\mathrm{N}$, total number of patients; $\mathrm{n}$, number of patients with events; P, placebo; RR, rate ratio; SD, standard deviation.

\section{Data Sharing Statement}

Data underlying the findings described in this manuscript, including individual deidentified participant data, protocols and clinical trial documents, may be obtained in accordance with AstraZeneca's data-sharing policy (described at https://astrazenecagrouptrials.pharmacm.com/ST/ $\underline{\text { Submission/Disclosure) through Vivli (https://vivli.org/). }}$

\section{Ethics Approval and Consent to Participate}

The study was performed in accordance with the Declaration of Helsinki and Good Clinical Practice
Guidelines. The trial protocol and informed consent procedures were approved by the institutional review board with controlling authority at each study site (see Supplementary Table 1 for further details). All participants provided written informed consent.

\section{Acknowledgments}

Editorial support, under the direction of the authors, was provided by Richard Knight, $\mathrm{PhD}$, and Sarah Hoyle, $\mathrm{PhD}$, CMC Connect, McCann Health Medical Communications, and funded by AstraZeneca in accordance with Good Publication Practice (GPP3) guidelines (Ann Intern Med. 2015;163:461-464).

\section{Author Contributions}

RAW had full access to all the data in the study and takes responsibility for the integrity of the data and the accuracy of the data analysis. All authors made a significant contribution to the work reported, whether that is in the conception, study design, execution, acquisition of data, analysis and interpretation, or in all these areas; took part in drafting, revising or critically reviewing the article; gave final approval of the version to be published; have agreed on the journal to which the article has been submitted; and agree to be accountable for all aspects of the work. 


\section{Funding}

The ASCENT-COPD study was initially funded by Forest Laboratories and later funded by AstraZeneca and Circassia. AstraZeneca was involved in data collection and interpretation, and the development and review of this manuscript. The decision to submit the manuscript for publication was made by the authors.

\section{Disclosure}

RAW reports personal fees from AstraZeneca during the conduct of the study; personal fees from AbbVie, Anaptsys Bio, AstraZeneca/MedImmune, Bristol Myers Squibb, Chimerix, Circassia, ContraFect, Galderma, GlaxoSmithKline, Kamada, Kinevant, Kiniksa, Merck, Novartis, Pneuma, Propeller Health, Pulmonx, Roche, Sunovion, and Verona, outside the submitted work; and research grants from AstraZeneca/MedImmune, Boehringer Ingelheim, and Pearl Therapeutics, outside the submitted work. KRC reports personal fees from AstraZeneca, Boehringer Ingelheim, CIHR-GSK Research Chair in Respiratory Health Care Delivery (UHN), CSL Behring, Genentech, Grifols, Kamada, Merck, Novartis, Roche, and Sanofi; and grants from Amgen, AstraZeneca, Bayer, Baxter, Boehringer Ingelheim, CSL Behring, Genentech, GlaxoSmithKline, Grifols, Kamada, Mereo Biopharma, Novartis, Regeneron, Roche, Sanofi, Shire, and Vertex, during the conduct of the study. BMS is a member of the TIMI Study Group which has received institutional research grant support through Brigham and Women's Hospital from Abbott, Amgen, Aralez, AstraZeneca, Bayer HealthCare Pharmaceuticals, Inc., BRAHMS, Daiichi-Sankyo, Eisai, GlaxoSmithKline, Intarcia, Janssen, MedImmune, Merck, Novartis, Pfizer, Poxel, Quark Pharmaceuticals, Roche, Takeda, The Medicines Company, and Zora Biosciences. He reports consulting fees from AbbVie, Allergan, AstraZeneca, Boehringer Ingelheim, Eisai, Elsevier Practice Update Cardiology, Esperion, Hamni, Lexicon, Medtronic, Merck, NovoNordisk, outside the submitted work; grants from Eisai, Merck, Novartis, NovoNordisk, and Pfizer Inc, outside the submitted work; and equity in Health[at]Scale. SZD and EG-G are employees of AstraZeneca. DL is an employee of PHASTAR and former consultant statistician to AstraZeneca. The authors report no other conflicts of interest in this work.

\section{References}

1. Global Initiative for Chronic Obstructive Lung Disease. Global strategy for the diagnosis, management, and prevention of chronic obstructive pulmonary disease. 2020. Available from: https://goldcopd.org/ wp-content/uploads/2019/11/GOLD-2020-REPORT-ver1.0wms.pdf. Accessed January 28, 2020.
2. Hoogendoorn M, Feenstra TL, Boland M, et al. Prediction models for exacerbations in different COPD patient populations: comparing results of five large data sources. Int J Chron Obstruct Pulmon Dis. 2017;12:3183-3194.

3. Lipson DA, Barnhart F, Brealey N, et al. Once-daily single-inhaler triple versus dual therapy in patients with COPD. $N$ Engl $J$ Med. 2018;378(18):1671-1680.

4. Donaldson GC, Seemungal TA, Bhowmik A, Wedzicha JA. Relationship between exacerbation frequency and lung function decline in chronic obstructive pulmonary disease. Thorax. 2002;57 (10):847-852.

5. Halpin DMG, Decramer M, Celli BR, Mueller A, Metzdorf N, Tashkin DP. Effect of a single exacerbation on decline in lung function in COPD. Respir Med. 2017;128:85-91.

6. Donaldson GC, Hurst JR, Smith CJ, Hubbard RB, Wedzicha JA. Increased risk of myocardial infarction and stroke following exacerbation of COPD. Chest. 2010;137(5):1091-1097.

7. Halpin DM, Decramer M, Celli B, Kesten S, Leimer I, Tashkin DP. Risk of nonlower respiratory serious adverse events following COPD exacerbations in the 4-year UPLIFT(R) trial. Lung. 2011;189(4):261-268.

8. Rothnie KJ, Yan R, Smeeth L, Quint JK. Risk of myocardial infarction (MI) and death following MI in people with chronic obstructive pulmonary disease (COPD): a systematic review and meta-analysis. BMJ Open. 2015;5(9):e007824.

9. Rothnie KJ, Connell O, Mullerova H, et al. Myocardial infarction and ischaemic stroke after exacerbations of chronic obstructive pulmonary disease. Ann Am Thorac Soc. 2018;15(8):935-946.

10. Suissa S, Dell'Aniello S, Ernst P. Long-term natural history of chronic obstructive pulmonary disease: severe exacerbations and mortality. Thorax. 2012;67(11):957-963. doi:10.1136/thoraxjnl2011-201518

11. Soler-Cataluña JJ, Martínez-García MA, Román Sánchez P, Salcedo E, Navarro M, Ochando R. Severe acute exacerbations and mortality in patients with chronic obstructive pulmonary disease. Thorax. 2005;60(11):925-931. doi:10.1136/thx.2005.040527

12. Rothnie KJ, Müllerová H, Smeeth L, Quint JK. Natural history of chronic obstructive pulmonary disease exacerbations in a general practice-based population with chronic obstructive pulmonary disease. Am J Respir Crit Care Med. 2018;198(4):464-471. doi:10.1164/ rccm.201710-2029OC

13. AstraZeneca UK Ltd. Eklira Genuair Summary of Product Characteristics. 2017. Available from: https://www.ema.europa.eu/ en/documents/product-information/eklira-genuair-epar-productinformation_en.pdf. Accessed July 16, 2020.

14. AstraZeneca PLC. Prescribing information Tudorza ${ }^{\circledR}$ Pressair $^{\circledR}$ (aclidinium bromide $400 \mu \mathrm{g}$ inhalation powder). 2016. Available from: http://www.accessdata.fda.gov/drugsatfda_docs/label/2016/ 202450s006lbl.pdf. Accessed June 3, 2016.

15. Wise RA, Chapman KR, Scirica BM, et al. Effect of aclidinium bromide on major cardiovascular events and exacerbations in high-risk patients with chronic obstructive pulmonary disease: the ASCENT-COPD randomized clinical trial. JAMA. 2019;321 (17):1693-1701. doi:10.1001/jama.2019.4973

16. Tashkin DP, Celli B, Senn S, et al. A 4-year trial of tiotropium in chronic obstructive pulmonary disease. $N$ Engl J Med. 2008;359 (15):1543-1554. doi:10.1056/NEJMoa0805800

17. Sethi S, Kerwin E, Watz H, et al. AMPLIFY: a randomized, Phase III study evaluating the efficacy and safety of aclidinium/formoterol vs monocomponents and tiotropium in patients with moderate-to-very severe symptomatic COPD. Int $J$ Chron Obstruct Pulmon Dis. 2019;14:667-682.

18. Wise RA, Chapman KR, Scirica BM, et al. Long-term evaluation of the effects of aclidinium bromide on major adverse cardiovascular events and COPD exacerbations in patients with moderate to very severe COPD: rationale and design of the ASCENT COPD study. Chronic Obstr Pulm Dis. 2018;5(1):5-15. 
19. Halpin DMG, Tashkin DP, Celli BR, Leimer I, Metzdorf N, Decramer M. Effect of tiotropium on outcomes in patients with COPD, categorized using the new GOLD Grading System: results of the UPLIFT(R) Randomized controlled trial. Chronic Obstr Pulm Dis. 2015;2(3):236-251.
20. Barnes N, Calverley PM, Kaplan A, Rabe KF. Chronic obstructive pulmonary disease and exacerbations: patient insights from the global Hidden Depths of COPD survey. BMC Pulm Med. 2013;13:54.

\section{Publish your work in this journal}

The International Journal of COPD is an international, peer-reviewed journal of therapeutics and pharmacology focusing on concise rapid reporting of clinical studies and reviews in COPD. Special focus is given to the pathophysiological processes underlying the disease, intervention programs, patient focused education, and self management protocols. This journal is indexed on PubMed Central, MedLine and CAS. The manuscript management system is completely online and includes a very quick and fair peer-review system, which is all easy to use. Visit http://www.dovepress.com/testimonials.php to read real quotes from published authors.

Submit your manuscript here: https://www.dovepress.com/international-journal-of-chronic-obstructive-pulmonary-disease-journal 